\title{
5 Der Mehrwert bisheriger Forschungen und das bestehende Desiderat
}

$\mathrm{Zu}$ der Problematik des Burnout-Syndroms, seiner Entstehung und seiner Auswirkungen sowie zu seiner Prävention sind in den letzten Jahren zahlreiche Studien und Untersuchungen entstanden, die bedeutende Erkenntnisse liefern:

- Die psychologischen Untersuchungen zeigen, dass der Diskurs um Stress deutlich mehr an Bedeutung gewonnen hat und dass die Verbindung gesunder psychischer, physischer und geistiger Zustände konstitutiv für das allgemeine Wohlbefinden des Menschen ist. Sie liefern wichtige Kenntnisse darüber, wie der menschliche Organismus die Stressreaktionen verarbeitet und wieso Ermüdung und Erschöpfung auf dauerhaften, unkontrollierbaren Stress folgen.

- Die arbeitssoziologischen Untersuchungen legen den Paradigmenwechsel in der Wahrnehmung der Arbeit nahe, welcher zur kontextuellen und indirekten Steuerung der Arbeitssubjekte geführt hat. Sie beleuchten, wie die veränderte Arbeitsorganisation und die steigenden Ansprüche an die Einzelnen und die Kollektive Strukturkonstellationen erzeugen, die zur Überforderung und zum Kontrollverlust führen.

- Die soziokulturellen Untersuchungen problematisieren das soziale Empfinden von Leiden und Erschöpfung und erforschen, wie sich das Bewusstsein von diesen Kategorien sozial verbreitet und konstitutiv für das Verständnis des modernen Subjekts wird. Insbesondere zeigen sie, welchen Belastungen durch die sozialkulturellen Entwicklungen die Subjekte zunehmend ausgesetzt sind und wie ihre Empfindungen - etwa auch das Burnout-Syndrom - sozial behandelt und verarbeitet werden.

- Die kulturgeschichtlichen Überlegungen zur Prävention des Leidens und Unbehagens des Einzelnen und der ganzen Gesellschaften weisen hin auf die wandelnde Empfindung des Leidens, das individuell, kollektiv und global spürbar wird. Vor allem zeigen sie die Spanne auf, in der sich die Prävention als Kulturtechnik und Zeitform etabliert und auf neue Herausforderungen reagiert hat.

Der aktuelle Kenntnisstand hilft dieser Untersuchung, das Phänomen des Burnout-Syndroms in seinen soziologischen und kulturellen Facetten in den westlichen, industriellen Gesellschaften aufzugreifen. Es zeigt außerdem die Argumentationslinien, entlang welchen sich die Diskussion um dieses soziale Phänomen bewegt, insbesondere die strukturellen Veränderungen in den Arbeitsprozessen, die Wandlung des Gesundheitsbegriffs und das wachsende Interesse an der Prävention. Schließlich verhilft der bisherige Kenntnisstand zu begreifen, wie das Wissen um das Burnout-Syndrom entsteht, etwa in Verbindung zur Depressi- 
on, zu den gegenwärtigen Arbeitsanforderungen oder zu den Ansprüchen an das moderne Individuum, das Leben selbstständig zu meistern und die Misserfolge als neue Erfolgschancen zu deuten.

Wofür der aktuelle Forschungsstand allerdings blind bleibt und damit auf eine Forschungslücke hinweist, ist die Art und Weise dessen, welche Auswirkungen und Effekte durch das Burnout-Syndrom begünstigt und in Form von präventiven Maßnahmen eingeleitet werden: Welche Art von gesellschaftlichen und individuellen Eingriffen wird unter dem Verweis auf Burnout-Prävention möglich? Wen und wozu legitimiert die Prävention von Burnout-Syndrom? Durch welche Mechanismen dringt sie als Selbstverständlichkeit in das Bewusstsein der Bevölkerung ein und wie wird sie gesellschaftlich akzeptabel gemacht? Mit welchen Machteffekten geht sie einher und wie (re-)strukturiert sie die gesellschaftlichen Machtund Herrschaftsverhältnisse? Welche Umdeutungen, diskursive Verschiebungen und Akzentuierungen entstehen dabei? Wie wird sie im Alltag als eine immanente Regierungsweise präsent? Welche gesellschaftskritischen Konsequenzen ließen sich daraus ziehen? Diese Schattenseite des Burnout-Syndroms hat bislang wenig Aufmerksamkeit bekommen, worauf die bestehende Untersuchung der BurnoutPrävention eingehen und den Forschungsstand damit um neue Kenntnisse und kritische Einsichten ergänzen möchte. 